T. Sasaki

Nagoya Math. J.

Vol. 71 (1978), 97-105

\title{
SOME EXAMPLES OF STOCHASTICALLY STABLE HOMEOMORPHISMS
}

\author{
TAKESHI SASAKI
}

\section{§ 0. Introduction}

Recently A. Morimoto [1] has proved the Takens conjecture in the tolerance stability by using the notion of pseudo-orbits and the stochastic stability. He also characterized group automorphisms of a torus to be stochastically stable and clarified the relations to other stabilities.

In this paper we shall give the condition for spherical or projective linear transformations to be stochastically stable.

The author would like to express his hearty thanks, to Professor A. Morimoto who taught him the problem and gave him advices for the completion of this paper.

\section{§1. Definitions and results}

Let $\phi: X \rightarrow X$ be a homeomorphism of a compact metric space $(X, d)$. A sequence $\left\{x_{i}\right\}$ of points $x_{i} \in X, i \in Z$, is called a $\delta$-pseudo orbit of $\phi$ if $d\left(\phi\left(x_{i}\right), x_{i+1}\right)<\delta$ holds for every $i \in Z$. We denote by $\operatorname{Orb}^{\delta}(\phi)$ the set of all $\delta$-pseudo orbits of $\phi$, and by $\overline{\operatorname{Orb}^{\delta}}(\phi)$ the set of all closed subsets of $X$ which are the closure of $\delta$-pseudo orbit of $\phi . O_{\phi}(x)=$ the closure of the orbit of $\phi$ through $x$.

Let $C(X)$ be the set of all non-empty closed sets in $X . \quad C(X)$ will be a compact metric space by the distance function $\bar{d}$ defined by

$$
\bar{d}(A, B)=\operatorname{Max}\left\{\operatorname{Max}_{b \in B} d(A, b), \operatorname{Max}_{a \in A} d(a, B)\right\},
$$

for $A, B \in C(X)$, where $d(A, b)=\inf _{a \in A} d(a, b)$. An element $A$ of $C(X)$ is called an extended orbit of $\phi$ iff for any $\varepsilon>0$ there is $A_{s} \in \overline{\operatorname{Orb}^{s}}(\phi)$ with $\bar{d}\left(A, A_{s}\right)<\varepsilon$. We denote by $E_{\phi}$ the set of all extended orbits of $\phi$, and $O_{\phi}=$ the closure of $\left\{O_{\phi}(x) \mid x \in X\right\}$ in $C(X)$.

Received June 20, 1977. 
Definition 1. A homeomorphism $\varphi$ is called $O E$ if $O_{\phi}=E_{\phi}$.

Given $\varepsilon>0$, a $\delta$-pseudo orbit $\left\{x_{i}\right\}$ is called to be $\varepsilon$-traced by a point $x \in X$ iff $d\left(\phi^{i}(x), x_{i}\right) \leqq \varepsilon$ for every $i \in Z$.

DEFINITION 2. $\phi$ is called stochastically stable (abbriv. $P O$ ) iff for any $\varepsilon>0$ there exists $\delta>0$ such that any $\delta$-pseudo orbit of $\phi$ can be $\varepsilon$-traced by some point $x \in X$.

Relating to these notions we have the following theorems.

THEOREM I ([1]). If $\phi$ is $P O$, then it is $O E$.

THEOREM II ([1]). If the space $X$ is a manifold and $\phi$ is a $C^{1}$-diffeomorphism satisfying Axiom $A$ and the strong transversality condition, then it is PO. Especially if $\phi$ is a Morse-Smale diffeomorphism, then it is $P O$.

Therefore by the celebrating theorem of Anosov

COROllary. If $\phi: X \rightarrow X$ is an Anosov diffeomorphism, it is PO.

Moreover we have

THEOREM III ([1]). Any isometry of a compact Riemannian manifold of positive dimension is not $P O$.

Now we shall state the results.

Let $\phi$ be a general linear transformation of $\boldsymbol{R}^{n+1}$, that is, a matrix $\phi \in G L(n+1, R)$. Then it induces on the sphere a diffeomorphism $\tilde{\phi}$ which is defined by

$$
\tilde{\phi}(x)=\frac{\phi(x)}{|\phi(x)|} \quad \text { for } x \in S^{n},
$$

where $|\cdot|$ is the euclidean norm. We call the transformation of this type a spherical linear transformation.

THEOREM 1. A spherical linear transformation $\tilde{\phi}$ is $P O$ iff the absolute value of the eigenvalues of the associated matrix $\phi$ are all mutually distinct.

Clearly $\phi$ induces the real projective linear transformation $\hat{\phi}^{\prime}$ of $P^{n}(R)$ given by

$$
\tilde{\phi}^{\prime}([x])=[\phi(x)]
$$


for $[x] \in P^{n}(\boldsymbol{R}),[x]$ being the line through $x$ and the origin of $\boldsymbol{R}^{n+1}$. Denoting by $\pi: S^{n} \rightarrow P^{n}(R)$ the natural projection, we have $\tilde{\phi}^{\prime} \circ \pi=\pi \circ \tilde{\phi}$. Therefore combining a result in [1] and Theorem 1 , we obtain

COROLlaRY 1. A real projective linear transformation $\tilde{\phi}^{\prime}$ is $P O$ iff the absolute value of the eigenvalues of the associated matrix $\phi$ are mutually distinct.

Similarly let $\psi$ be an element of $G L(n+1, C)$. By $\tilde{\psi}$ we denote the associated projective linear transformation on $P^{n}(C)$. The we shall prove

THEOREM 2. $\tilde{\psi}$ is PO iff the absolute value of eigenvalues of $\psi$ are all mutually distinct.

$\psi$ also induces a transformation $\hat{\psi}$ on $S^{2 n+1} \subset C^{n+1}$ as in the real case. But for $\hat{\psi}$ we get

COROllary $3 . \quad \hat{\psi}$ is not $P O$.

\section{§2. Spherical linear transformations}

Let $\phi($ resp. $\psi$ ) be a real non-singular matrix of size $n+1$, and $\tilde{\phi}$ (resp. $\tilde{\psi}$ ) the induced spherical transformation of $S^{n} . S^{n}$ is endowed with the canonical distance function $d_{n}$. We can easily verify that $\widetilde{\phi} \circ \psi=\tilde{\phi} \circ \tilde{\psi}$ and hence, by the following Lemma 1, we see that if $\phi$ and $\psi$ are conjugate then $\tilde{\phi}$ is $P O$ if and only if $\tilde{\psi}$ is.

LEMMA 1 ([1]). Let $h_{1}, h_{2}$ be homeomorphisms of a compact metric space, and set $h_{3}=h_{2} \circ h_{1} \circ h_{2}^{-1}$. Then $h_{1}$ is PO iff $h_{3}$ is.

LEMMA 2. Let $\phi$ be reducible of type $\left(\begin{array}{ll}\phi_{1} & 0 \\ * & *\end{array}\right), \phi_{1} \in G L(m+1, R)$, $m<n$. If $\tilde{\phi}$ is PO, then $\tilde{\phi}_{1}$ is PO.

Proof. For $x=\left(x_{0}, \cdots, x_{n}\right) \in \boldsymbol{R}^{n+1}$ set $x^{\prime}=\left(x_{0}, \cdots, x_{m}\right), x^{\prime \prime}=\left(x_{m+1}\right.$, $\left.\cdots, x_{n}\right)$. Define $S^{m}=\left\{x \in S^{n} \mid x^{\prime \prime}=0\right\}$ and $P=\left\{x \in S^{n} \mid x^{\prime}=0\right\}$. We can define the projection $\pi: S^{n}-P \rightarrow S^{m}$ by $\pi(x)=\frac{1}{\left|x^{\prime}\right|} x^{\prime} . \quad \pi$ is distance decreasing in the following sense, i.e. $d_{n}(x, y) \geq d_{m}(\pi(x), y)$ holds for $x \in S^{n}, y \in S^{m}$. By the definition $\pi \tilde{\phi}(x)=\tilde{\phi}_{1} \pi(x)$ for $x \in S^{n}-P$. To prove $\tilde{\phi}_{1}$ is $P O$, fix $\varepsilon>0$. Here we may assume $\varepsilon<\bar{d}\left(S^{m}, P\right)$. Since $\tilde{\phi}$ is $P O$, 
there exists $\delta>0$ for this $\varepsilon$ such that every $\delta$-pseudo orbit of $\tilde{\phi}$ is $\varepsilon$ traced. Let $\left\{x_{i}\right\}_{i \in Z}, x_{i} \in S^{m}$, be a $\delta$-pseudo orbit of $\tilde{\phi}_{1}$. Since $\left\{x_{i}\right\}$ is also a $\delta$-pseudo orbit of $\tilde{\phi}$, this can be $\varepsilon$-traced by some point $x \in S^{n}: d_{n}\left(\phi^{i}(x)\right.$, $x_{i}$ ) $\leqq, i \in Z$. Therefore by the distance decreasing property of $\pi$ as mentioned above we have $\varepsilon \geqq d_{m}\left(\pi \tilde{\phi}^{i}(x), x_{i}\right)=d_{m}\left(\tilde{\phi}_{1}^{i} \pi(x), x_{i}\right)$, which says that $\left\{x_{i}\right\}$ is $\varepsilon$-traced by $\pi(x)$. Hence $\tilde{\phi}_{1}$ is $P O$.

LEMMA 3. If $\phi$ is a matrix of the form $\left[\begin{array}{lllll}\lambda & 1 & & & \\ \cdot & \cdot & \cdot & & \\ & & & & 1 \\ & & & & \lambda\end{array}\right]$ of size $n+1 \geqq 2, \tilde{\phi}$ is not $P O$.

Proof. By Lemma 1 and the fact that $\phi$ and $c \phi(c \neq 0 \in R)$ induce the same spherical transformation we can assume $\lambda=1$. Then by a simple calculation

$$
\begin{aligned}
\tilde{\phi}^{k}(x) & =\frac{1}{\left|y_{k}\right|} y_{k}, \\
y_{k} & =\left(\sum_{j=0}^{n}\left(\begin{array}{c}
k \\
j
\end{array}\right) x_{j}, \cdots, \sum_{j=0}^{i}\left(\begin{array}{c}
k \\
j
\end{array}\right) x_{j}, \cdots, x_{n}\right) .
\end{aligned}
$$

Hence (1) if $x_{n} \geqq 0$ (resp. $\left.\leqq 0\right)$ then $\left(\tilde{\phi}^{k}(x)\right)_{n} \geqq 0$ (resp. $\leqq 0$ ) and (2) $\tilde{\phi}^{k} x$ $\rightarrow(1,0, \cdots, 0)$ (resp. $(-1,0, \cdots, 0)$ ) if $k \rightarrow+\infty$ and $x_{n}>0$ or $k \rightarrow-\infty$ and $x_{n}<0$ (resp. $k \rightarrow+\infty$ and $x_{n}<0$ or $k \rightarrow-\infty$ and $x_{n}>0$ ). To prove $\tilde{\phi}$ is not $P O$, it is enough to find $\varepsilon>0$ and a $\delta$-pseudo orbit for any $\delta>0$ which cannot be $\varepsilon$-traced. But this is achieved by the properties (1) and (2). In fact, by (2) we can construct, for any $\delta>0$, a $\delta$-pseudo orbit combining the upper hemisphere and the lower one, but (1) means every orbit stays always in the same hemisphere.

LEMMA 4. Let $\phi=\left(\begin{array}{llll}R_{\theta} & I_{2} & & \\ \cdot & \cdot & \cdot & \\ & \cdot & \cdot & \\ & & I_{2}\end{array}\right)$, where $R_{\theta}=\left(\begin{array}{rr}\cos \theta & -\sin \theta \\ \sin \theta & \cos \theta\end{array}\right)$, $I_{2}=\left(\begin{array}{ll}1 & 0 \\ 0 & 1\end{array}\right)$. Then $\tilde{\phi}$ is not PO.

Proof. In case $\phi=R_{\theta}, \tilde{\phi}$ is not $P O$ because $\tilde{\phi}$ is an isometry (cf. Theorem III). In case the size of $\phi$ is not smaller than 4, for the sake of simplicity, we shall prove this Lemma for $\phi=\left(\begin{array}{cc}R_{\theta} & I_{2} \\ & R_{\theta}\end{array}\right)$. In this 
case, introducing new variables $u=x_{0}+\sqrt{-1} x_{1}$ and $v=x_{2}+\sqrt{-1} x_{3}$, we have

$$
\left\{\begin{array}{l}
\phi^{n}(u, v)=e^{i n \theta}\left(u_{n}, v_{n}\right) \\
u_{n}=u+n e^{-i \theta} v, \quad v_{n}=v .
\end{array}\right.
$$

Therefore

(1) Every orbit approaches to $S^{1}=\left\{(u, v) \in S^{3} \mid v=0\right\}$ in the limit of both directions, and

(2) $\tilde{\phi} \mid S^{1}$ is a rotation. Hence there exists a $\delta$-pseudo orbit of $\tilde{\phi} \mid S^{1}$ for any $\delta>0$ which is dense in $S^{1}$. By (1) and (2) we can easily construct a dense $\delta$-pseudo orbit of $\tilde{\phi}$. Hence $E_{\tilde{\phi}} \ni S^{3}$. On the other hand, for some small neighbourhood $U$ of $(u, v)=(0,1)$, there exists a positive constant $c$ depending only on $U$ such that $d(\tilde{\phi}(x), x) \geqq c$ and $\phi^{k}(x)$ $\notin U, k \neq 0$, for $x \in U$. Therefore $O_{\tilde{\phi}} \notin S^{3}$. Hence $\tilde{\phi}$ is not $O E$. By Theorem I $\tilde{\phi}$ is not $P O$.

Proof of Theorem 1. Assume $\tilde{\phi}$ is PO. By the remark preceding Lemma 1 the transformation associated with the Jordan canonical form of $\phi$ is also $P O$. By Lemma 2 each block gives a $P O$ transformation. Then by Lemma 3 and 4 each block must be of size 1 . Therefore, by making use of Lemma 2 again, we see that all eigenvalues of $\phi$ are real and mutually distinct. Moreover $\phi$ does not contain a component of type $\left(\begin{array}{rr}\lambda & 0 \\ 0 & -\lambda\end{array}\right)$, because $\left(\begin{array}{rr}\lambda & 0 \\ 0 & -\lambda\end{array}\right)$ is not $P O$ by Theorem III. Hence all eigenvalues of $\phi$ are mutually distinct in absolute value.

Conversely let $\phi=\left(\begin{array}{lll}\lambda_{0} & & \\ & \cdot & \\ & & \cdot \\ & & \lambda_{n}\end{array}\right)$ and we may assume $\left|\lambda_{i}\right|>\left|\lambda_{j}\right|$ for $0 \leq i<j \leq n$. Then the periodic point set of $\tilde{\phi}$ is $\left\{p_{i}^{ \pm} \mid 0 \leq i \leq n\right\}$, where $p_{i}^{ \pm}=(\overbrace{0, \cdots, 0}^{i}, 1,0, \cdots, 0)$. If we identify $T_{x} S^{n}$ with the set $\left\{y \in \boldsymbol{R}^{n+1}\right.$ $\left.x_{0} y_{0}+\cdots+x_{n} y_{n}=0\right\}$, then $\phi_{*} y=\left(\frac{\lambda_{0}}{\lambda_{i}} y_{0}, \cdots, \frac{\lambda_{i-1}}{\lambda_{i}} y_{i-1}, 0, \frac{\lambda_{i+1}}{\lambda_{i}} y_{i+1}, \cdots, \frac{\lambda_{n}}{\lambda_{i}} y_{n}\right)$ for $y \in T_{p_{i}^{ \pm}}\left(S^{n}\right)$. Therefore $\tilde{\phi}$ is hyperbolic at $p_{i}^{ \pm}, 0 \leq i \leq n$. Moreover we see that the stable manifold $W^{s}\left(p_{i}^{ \pm}\right)$at $p_{i}^{ \pm}$is the set $\left\{x \in S^{n} \mid x_{0}=\cdots\right.$ $\left.=x_{i-1}=0, x_{i}>0\right\}$ and the unstable manifold $W^{u}\left(p_{j}^{ \pm}\right)$at $p_{j}^{ \pm}$is the set $\left\{x \in S^{n} \mid x_{j}>0, x_{j+1}=\cdots=x_{n}=0\right\}$, both in the case that $\lambda_{0}>\cdots>\lambda_{n}$ $>0$. Hence $W^{s}\left(p_{i}^{ \pm}\right)$and $W^{u}\left(p_{j}^{ \pm}\right)$have only transversal intersection. 
Since $W^{s}(x)=\emptyset$ and $W^{u}(x)=\emptyset$ for $x \neq p_{i}^{ \pm}, \tilde{\phi}$ satisfies the strong transversality condition. When the sign of $\lambda_{i}$ is in the other case we can see the same property. This means, namely, that $\tilde{\phi}$ is a Morse-Smale diffeomorphisms, especially $P O$ by Theorem II.

COROLlaRY 2. Let $\tilde{\phi}$ be a spherical linear transformation. Then the following conditions for $\tilde{\phi}$ are mutually equivalent:

(1) $\tilde{\phi}$ is stochastically stable $(P O)$,

(2) $\tilde{\phi}$ is a Morse-Smale diffeomorphism,

(3) $\tilde{\phi}$ satisfies Axiom $A$ and the strong transversality condition,

(4) $\tilde{\phi}$ is topologically stable.

Proof. (1) $\rightarrow$ (2) is shown in the proof of Theorem 1.

$(2) \rightarrow(3) \rightarrow(1)$ is by Theorem II,

$(3) \rightarrow(4)$ is by Nitecki [2].

$(4) \rightarrow(1)$ is proved by Morimoto [1].

Let $\psi$ be an element in $G L(n+1, C)$. $\psi$ defines a transformation $\hat{\psi}: S^{n+1} \rightarrow S^{2 n+1}$ by $\hat{\psi}(x)=\frac{\psi(x)}{|\psi(x)|}$. If we consider $G L(n+1, C)$ as a subgroup of $G L(2 n+2, R)$ by the identification $\psi \leftrightarrow\left(\begin{array}{rr}\psi_{1} & -\psi_{2} \\ \psi_{2} & \psi_{1}\end{array}\right)=\psi^{\prime}$, where $\psi_{1}=\operatorname{Re} \psi$ and $\psi_{2}=\operatorname{Im} \psi$, then $\hat{\psi}$ is nothing but the spherical linear transformation $\hat{\psi}^{\prime}$ associated with $\psi^{\prime}$.

COROLlaRY 3. The transformation $\hat{\psi}$ cannot be PO.

Proof. Let $\lambda$ be a real eigenvalue of $\psi^{\prime}$ and $\left(\begin{array}{l}u \\ v\end{array}\right)$ be a corresponding eigenvector: $\psi^{\prime}\left(\begin{array}{l}u \\ v\end{array}\right)=\lambda\left(\begin{array}{l}u \\ v\end{array}\right)$. Then $\psi(u+\sqrt{-1} v)=\lambda(u+\sqrt{-1} v)$. Hence $\lambda$ is also an eigenvalue of $\psi$. Therefore, if $\hat{\psi}$ is $P O$, i.e. if $\psi^{\prime}$ has $2 n$ distinct real eigenvalues, then $\psi$ has also $2 n$ distinct eigenvalues. But this is a contradiction. Hence $\hat{\psi}$ cannot be $P O$.

\section{§3. Projective linear transformations}

We shall prove Theorem 2 along the same line as in the proof of Theorem 1.

Let $\psi$ be a matrix in $G L(n+1, C)$ and $\tilde{\psi}$ the associated element in $P G L(n+1, C) . \quad \tilde{\psi}$ is a projective linear transformation of $P^{n}(C)$. We 
denote by $z=\left[z_{0}, \cdots, z_{n}\right]$ a point of $P^{n}(C)$ in the homogeneous coordinate.

LEMMA $2^{\prime}$. Assume $\psi$ is reducible: $\psi=\left(\begin{array}{cc}\psi_{1} & 0 \\ * & *\end{array}\right)$ and the size of $\psi_{1}$ is $m+1$. Let $\boldsymbol{P}^{m}(\boldsymbol{C})=\left\{z \in P^{n}(C) \mid z_{m+1}=\cdots=z_{n}=0\right\}$. $\psi_{1}$ induces $a$ projective linear transformation $\tilde{\psi}_{1}$ on $P^{m}(C)$. Then $\tilde{\psi}_{1}$ is $P O$ if $\tilde{\psi}$ is.

Proof. Define the projection $\pi: P^{n}(C)-P \rightarrow P^{m}(C)$ by $\pi([z])=\left[z_{0}\right.$, $\left.\cdots, z_{m}\right]$, where $P:=\left\{z \in P^{n}(C) \mid z_{0}=\cdots=z_{m}=0\right\}$ is the pole of $\pi$. In this situation the proof is the same as that of Lemma 2 .

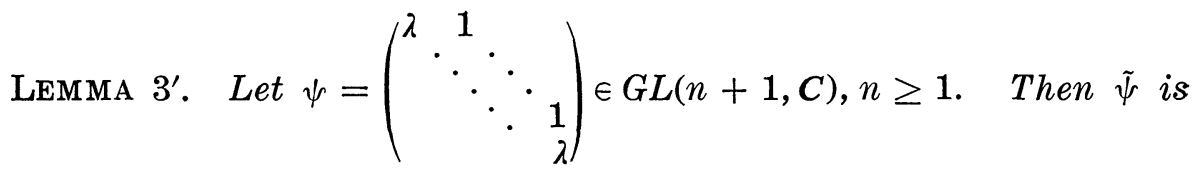
not $P O$.

Proof. By the same reason as in the proof of Lemma 3 we can assume $\lambda=1$. Let $P^{n-1}(C)=\left\{z \in P^{n}(C) \mid z_{n}=0\right\}$. Since we have

$$
\tilde{\psi}^{k}(z)=\left[z_{0}+k z_{1}+\frac{k(k-1)}{2}+\cdots, \cdots, z_{n-1}+k z_{n}, z_{n}\right],
$$

every orbit of $\tilde{\psi}$ approaches to $P^{n-1}(C)$ as $|k| \rightarrow \infty$, and $\tilde{\psi}$ leaves $P^{n-1}(C)$ invariant. First we show $E_{\tilde{\psi}} \ni P^{n}(C)$, by induction on $n$. If $n=1$, the orbit of $\tilde{\psi}$ approaches to one point (point at infinity). By the same argument as in the proof of Lemma 3 we have $E_{\tilde{\psi}} \ni P^{1}(C)$. For a general $n$, using the induction hypothesis on $P^{n-1}(C)$, we can construct a dense $\delta$-pseudo orbit for any $\delta>0$ by the above remark. Hence $E_{\tilde{\psi}}$ ə $P^{n}(C)$.

On the other hand, by the similar method as in the last part of the proof of Lemma 4 , we see that $\tilde{\psi}$ goes away uniformly in the neighbourhood of $[0, \cdots, 0,1]$. Hence, by the same reason as in the proof of Lemma $4, O_{\tilde{\psi}} \nexists P^{n}(C)$. Therefore $\tilde{\psi}$ is not $O E$, hence not $P O$.

Proof of Theorem 2. Assume $\tilde{\psi}$ is $P O$. By Lemmas $1,2^{\prime}$ and $3^{\prime}$ it follows that the absolute value of eigenvalues of $\psi$ are mutually distinct. Converse implication does hold by the same sort of reasoning as that for Theorem 1.

Similarly as Corollary 2, we have 
COROLlaRY 4. Let $\tilde{\psi} \in P G L(n+1, C)$. The following conditions for $\tilde{\psi}$ are equivalent:

(1) $\tilde{\psi}$ is stochastically stable $(P O)$,

(2) $\tilde{\psi}$ is a Morse-Smale diffeomorphism,

(3) $\tilde{\psi}$ satisfies Axiom $A$ and the strong transversality condition,

(4) $\tilde{\psi}$ is topologically stable.

\section{§4. Remark on group automorphisms of the $n$-torus $T^{n}$}

In [1] the relations among the stochastic stability and other stabilities are clarified for group automorphisms of $T^{n}$. Here we shall add the relation of the stochastic stability to ergodicity.

Proposition. Let $A \in S L(n, Z)$ be a group automorphism of $T^{n}$. If $A$ is $O E$, then it is ergodic with respect to the canonical measure on $T^{n}$.

Proof. Assume $A$ is not ergodic. It is classical that, for some integer $p \neq 0, A^{p}$ has 1 as an eigenvalue. Hence there exists a nonzero rational vector $u$ such that $\left({ }^{t} A^{k p}-I\right) u=0, k \in Z$. Let $H$ be the hyperplane in $\boldsymbol{R}^{n}$ orthogonal to $u: H=\{v \mid\langle v, u\rangle=0\}$. Since $u$ is rational, $H$ projects into the closed submanifold in $T^{n}$. But, for every $s \in Z,{ }^{t} A^{s}\left({ }^{t} A^{k p}-I\right) u=0$, it follows that $A^{k p+s} x \in A^{s} x+H$ for every $x \in T^{n}$. Hence $A^{N} x \in \bigcup_{s=0}^{p-1}\left(A^{s} x+H\right)=: U(x)$, for any $N \in Z . \quad U(x)$ is obviously closed and invariant under $A$. Therefore $O_{A} \rightarrow T^{n}$. However $E_{A} \ni U^{n}$ because the periodic points of $A$ is dense in $T^{n}$. Hence $A$ is not $O E$.

COROLlaRY 5. In case $n=1,2$ or 3 , the following conditions for the group automorphism $A$ of the torus $T^{n}$ are equivalent:

(1) $A$ is stochastically stable (PO),

(2) $A$ is $O E$,

(3) $A$ is ergodic.

Proof. (1) $\rightarrow$ (2) is Theorem I. (2) $\rightarrow$ (3) is Proposition. (3) $\rightarrow$ (1) follows from the fact that if some eigenvalue of $A$ is of absolute value one, then $A$ has a root of unity as an eigenvalue in case $n=1,2$ or 3. 


\section{REFERENCES}

[1] A. Morimoto, Stochastically stable diffeomorphisms and Takens conjecture, Kokyuroku of Research Institute for Mathematical Science, Kyoto Univ., 303 (1977) with the title "Local dynamical systems", pp. 8-24.

[ 2 ] Z. Nitecki, On semi-stability for diffeomorphisms, Inv. Math., 14 (1971), 83-122.

Department of Mathematics

Nagoya University 